\title{
Magneto-optical Kerr rotation spectra in Fe ultrathin film on noble metals
}

\author{
M. Hayashi ${ }^{a}$, T. Katayama ${ }^{b}$, Y. Suzuki ${ }^{b}$, M. Taninaka ${ }^{a}$, A. Thiaville ${ }^{c}$ and W. Geerts ${ }^{d}$ \\ ${ }^{a}$ College of Science \& Technology, Nihon University, Funabashi, Chiba 274, Japan \\ ${ }^{b}$ Electrotechnical Laboratory, Umezono 1-1-4, Tsukuba, Ibaraki 305, Japan \\ ' Université de Paris Sud, Lab. de Physique des Solids, 91405 Orsay, France \\ ${ }^{d}$ University of Twente, MESA Institute, 7500 AE Enschede, The Netherlands
}

We report on the Kerr effects of ultrathin Fe films on $\mathrm{Au}$ or $\mathrm{Ag}(100)$ substrates. In 3.5-4.5 eV, a new $\phi_{\mathrm{K}}$ peak appears only in $\mathrm{Fe} / \mathrm{Au}$. The $\epsilon_{x y}$ of Fe film below $8 \AA$ deposited on $\mathrm{Ag}$ is different from that of bulk, and shows some structures in $2-3 \mathrm{eV}$. This is thought to be due to polarized Au atoms adjacent to Fe layer.

\section{Introduction}

It is supposed that an ultrathin film has an electronic structure which is different from the bulk state. So far many theoretical and experimental works have been done on the electronic structures of ultrathin films [1]. However only a few works have been reported in the field of the magneto-optical effect of thin ferromagnetic films except for SMOKE [2]. Recently, we found a new magneto-optical Kerr rotation peak in $\mathrm{Fe}$ ultrathin films below $8 \AA$. These films were deposited on Au buffer layers [3]. Himpsel found evidence for the existence of quantum well states in Fe ultrathin films deposited on Au using inverse photoemission spectroscopy (IPES) $[4,5]$.

In this experiment, we prepared several kinds of $\mathrm{Fe}$ ultrathin films deposited on $\mathrm{Ag}(100)$ buffer layers. The magneto-optical Kerr rotation $\left(\phi_{\mathrm{K}}\right)$ spectra of these films were measured and compared with those deposited on Au buffer layers.

\section{Experimental procedures}

All the samples were deposited by means of a molecular beam epitaxy (MBE) technique using electron gun sources for $\mathrm{Fe}$ and $\mathrm{Au}$ and a Knudsen cell for Ag. During the deposition, the vacuum in the MBE chamber was better than $10^{-10}$ Torr. A (100) cleaved $\mathrm{MgO}$ single crystal was used as a substrate. After a thermal flashing of the substrate at $900^{\circ} \mathrm{C}$, an fcc $\mathrm{Ag}(100)$ layer of $2000 \AA$ was deposited on the substrate at room temperature (RT). This $\mathrm{Ag}$ film was annealed at $450^{\circ} \mathrm{C}$ for 1 minute. After cooling down to RT, a bcc

Correspondence to: Dr. Toshikazu Katayama, Electrotechnical Laboratory, Umezono 1-1-4, Tsukuba-shi, Ibaraki 305, Japan. Tel: 0298-58-5426 or 5433, Fax: 0298-58-5434.
Fe(100) layer was deposited on it and covered by an Au layer of $20 \AA$. The thicknesses of $\mathrm{Au}$ and $\mathrm{Fe}$ films were measured by a quartz thickness monitor and controlled by a shutter system. The growth modes were monitored by observation of reflective high energy electron diffraction (RHEED) patterns.

The $\phi_{\mathrm{K}}$ spectra at RT were measured by a Kerr rotation spectrometer in the photon energy range from 1.55 to $5.3 \mathrm{eV}$. The angle of incidence was 10 degree from the film surface normal. The Kerr ellipticity $\left(\eta_{K}\right)$ spectra were calculated from the value of $\phi_{K}$ by using the Kramers-Kronig $(\mathrm{K}-\mathrm{K})$ relation $[3,6]$. We calculated off-diagonal elements of the dielectric tensor $\left(\epsilon_{x y}\right)$ in Fe layers using the values of diagonal elements of the dielectric tensor $\left(\epsilon_{x x}\right)$ from the literature $[7,8]$ under the assumption of no $\epsilon_{x y}$ in noble metal layers.

\section{Results}

Fig. 1 shows the changes of the $\phi_{K}$ spectra as a function of $\mathrm{Fe}$ layer thickness $(d)$ in $\mathrm{Au}(20 \AA) / \mathrm{Fe}$ $(d \AA) / \mathrm{Au}(2000 \AA) / \mathrm{Ag}(2000 \AA) / \mathrm{MgO}(100)$ films. The vertical axis means a normalized value of $\phi_{\mathrm{K}}$ by the $\mathrm{Fe}$ film thickness. A large peak, which is a plasma enhancement due to the Au buffer layer, is observed at $2.5 \mathrm{eV}$. A new $\phi_{\mathrm{K}}$ peak appears in $3.5-4.5 \mathrm{eV}$ for $\mathrm{Fe}$ films thinner than $10 \AA$. This peak shifts toward higher energies with increasing $\mathrm{Fe}$ thickness.

In fig. 2(a), (b) the changes of $\phi_{\mathrm{K}}$ and $\eta_{\mathrm{K}}$ spectra as a function of $d$ in $\mathrm{Au}(20 \AA) / \mathrm{Fe}(d \AA) / \mathrm{Ag}(5000 \AA) /$ $\mathrm{MgO}(100)$ films are shown. A negative large enhanced $\phi_{\mathrm{K}}$ peak is also observed at $3.8 \mathrm{eV}$. We cannot confirm a new peak in 3.5-4.5 eV contrary to the Au cases. But, there seem to exist in the $2-3 \mathrm{eV}$ range some $\phi_{\mathrm{K}}$ structures for $\mathrm{Fe}$ layers thinner than $8 \AA$. Such kinds of $\phi_{\mathrm{K}}$ structures were not observed in the Fe layer above $10 \AA$. 


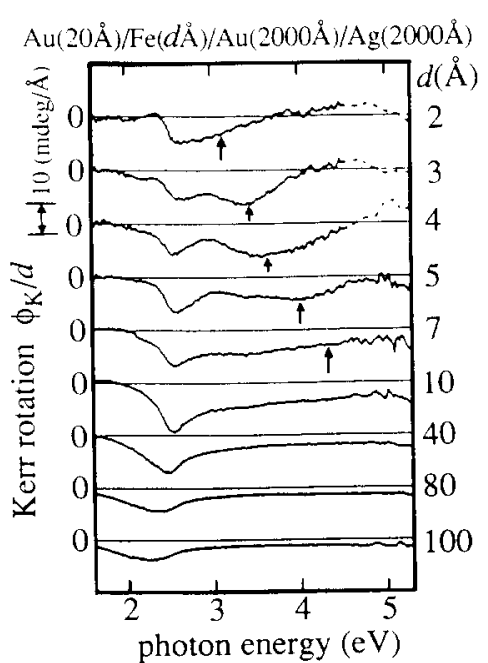

Fig. 1. Magneto-optical polar Kerr rotation $\left(\phi_{K}\right)$ spectra as a function of $\mathrm{Fe}$ layer thickness $(d)$ in $\mathrm{Au}(20 \AA) / \mathrm{Fe}(d \AA)$ / $\mathrm{Au}(2000 \AA) / \mathrm{Ag}(2000 \AA) / \mathrm{MgO}(100)$ films at room temperature. The vertical axis means values of $\phi_{K}$ normalized by the Fe film thickness.

In fig. 3(a), (b) are shown the spectra of the real part $\left(\epsilon_{x y}^{\prime}\right)$ and the imaginary part $\left(\epsilon_{0 y y}^{\prime \prime}\right)$ as a function of $d$ in $\mathrm{Au}(20 \AA) / \mathrm{Fe}(d \AA) / \mathrm{Ag}(5000 \AA) / \mathrm{MgO}(100)$ films. The $\epsilon_{x y}^{\prime}$ and $\epsilon_{x y}^{\prime \prime}$ spectra of bulk Fe [9] are shown as a comparison. The $\epsilon_{x y}$ structures near $3.8 \mathrm{eV}$ are thought to be due to an analytical error caused by errors of the optical constants of $\mathrm{Ag}$ [7]. The whole shape of the $\epsilon_{x y}^{\prime}$

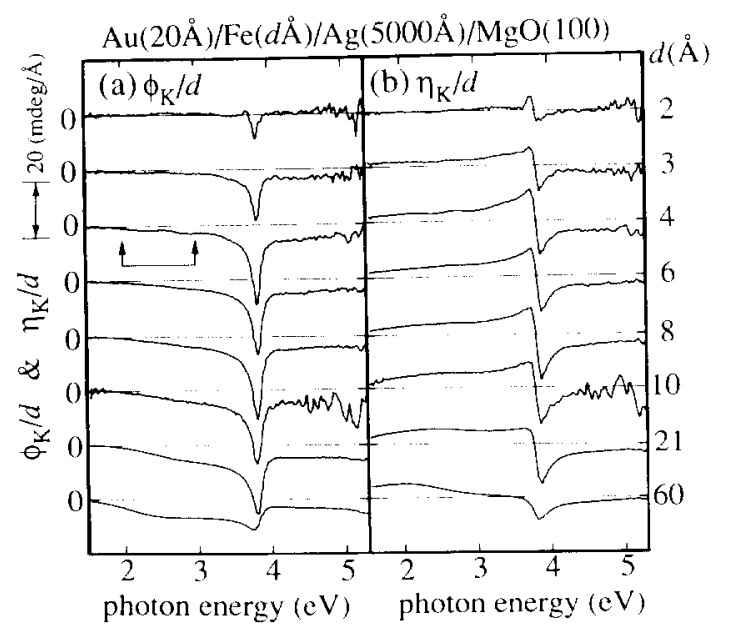

Fig. 2. Magneto-optical polar Kerr spectra as a function of $\mathrm{Fe}$ layer thickness $(d)$ in $\operatorname{Au}(20 \AA) / \operatorname{Fe}(d \AA) / \operatorname{Ag}(5000 \AA) /$ $\mathrm{MgO}(100)$ films at room temperature. (a) Kerr rotation $\left(\phi_{\mathrm{K}}\right)$ spectra, (b) Kerr ellipticity $\left(\eta_{K}\right)$ spectra. The vertical axis means values of $\phi_{\mathrm{K}}$ and $\eta_{\mathrm{K}}$ normalized by Fe film thickness.

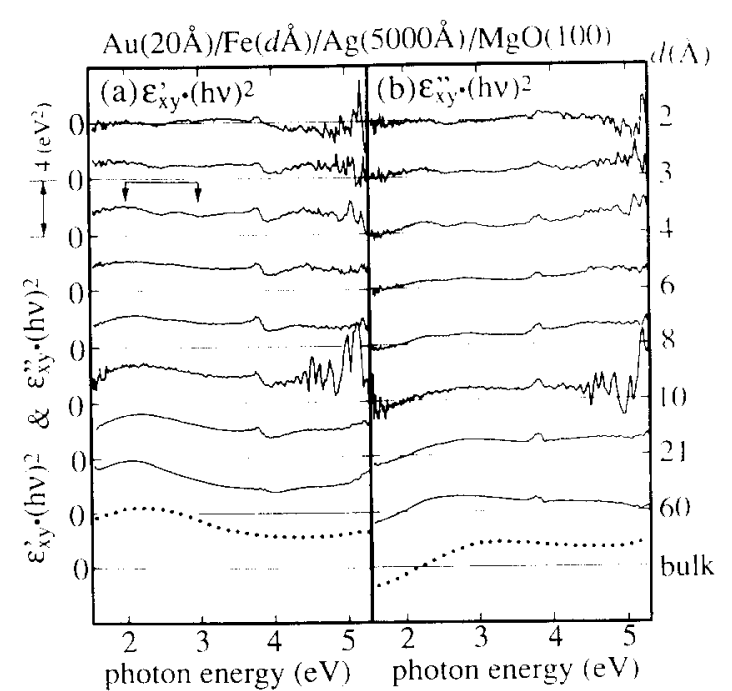

Fig. 3. Changes of the spectra of off-diagonal elements $\left(\epsilon_{,}\right)$ of the dielectric tensor as a function of Fe layers thickness $(d)$ in $\mathrm{Au}(20 \AA) / \mathrm{Fe}(d \stackrel{\mathrm{a}}{\mathrm{A}}) / \mathrm{Ag}(5000 \AA \mathrm{A}) / \mathrm{MgO}(100)$ films. The spectra of $F e$ bulk are shown as a comparison. (a) real part of $\epsilon_{1,},\left(\right.$ b) imaginary part of $\epsilon_{1}$.

spectrum has a tendency to approach towards that of bulk $\mathrm{Fe}$ with increasing $\mathrm{Fe}$ thickness. In the $2-3 \mathrm{eV}$ range, there are some structures of $\epsilon_{x y}$ in Fe films thinner than $8 \AA$. We measured an $\epsilon_{x y}^{\prime}$ change as a function of the $\mathrm{Au}$ layer thickness $(x)$ in $\operatorname{Au}(20 \AA) / \operatorname{Ag}(10 \AA) / \operatorname{Fe}(3 \AA) / \operatorname{Au}(x \AA) / \operatorname{Ag}(4000(0) /$ $\mathrm{MgO}(100)$ films to investigate the effect of the $A u$ layers. As a result, it is found that the $\epsilon_{x}^{\prime}$ structure near $2-3 \mathrm{eV}$ varies with changing $x$.

\section{Discussion}

As shown in fig. 1, a new $\phi_{\mathrm{K}}$ peak is observed. This peak shifts to higher encrgies with increasing Fe thickness in Fe ultrathin film deposited on Au. The phenomenon is thought to be due to a formation of quantum well states in Fe ultrathin films $[3,6]$. In the case of an $\mathrm{Ag}$ buffer layer, the $\phi_{k}$ peak does not appear at the same photon energy region. As a possible reason why the new $\phi_{K}$ peak did not appear in the Fc layer deposited on Ag, we consider the following two reasons: (1) The influence of the large $\phi_{K}$ enhancement by the plasma edge of $\mathrm{Ag}$. (2) The difference of crystal growth.

One of the reasons is that, since there is a large effect from $\phi_{\mathrm{K}}$ enhancement duc to the plasma edge of $\mathrm{Ag}$ near $3.5-4.5 \mathrm{eV}$, the appearance of a new peak may hardly be observed. It is found that this has not such a large effect upon the phenomenon from 


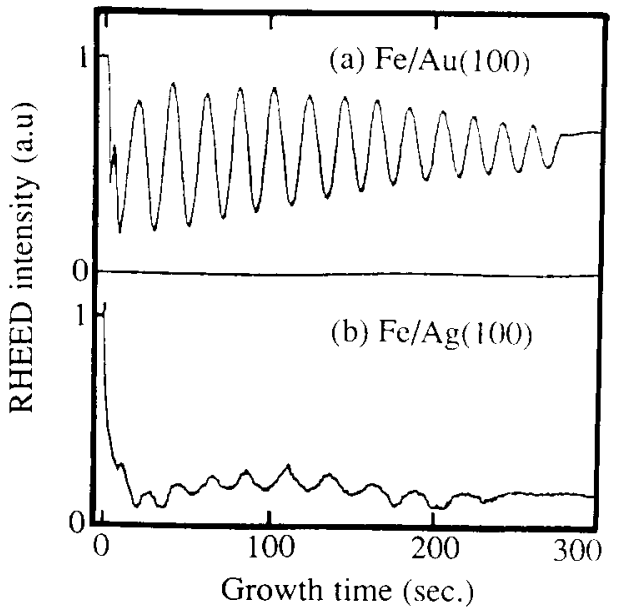

Fig. 4. The RHEED intensity oscillations during the growth of Fe layer deposited on (a) $\mathrm{Au}(100)$ and (b) $\mathrm{Ag}(100)$ buffer layers.

the results of difference of $\phi_{\mathrm{K}}$ spectra between $\mathrm{Au}(20 \AA) / \mathrm{Fe}(3 \AA) / \mathrm{Au}(2000 \AA) / \mathrm{Ag}(2000 \AA) / \mathrm{MgO}$ and $\operatorname{Au}(20 \AA) / \operatorname{Fe}(3 \AA) / \operatorname{Ag}(10 \AA) / \operatorname{Au}(2000 \AA) /$ $\operatorname{Ag}(2000 \AA) / \mathrm{MgO}$ films [6]. Next, we investigated the influence of the difference of growth modes between $\mathrm{Fe}$ layers on $\mathrm{Au}$ and $\mathrm{Ag}$ buffer layers. In fig. 4 the RHEED intensity oscillations during the growth of the Fe layer on $\mathrm{Au}$ and $\mathrm{Ag}(100)$ surfaces are shown. In the case of a Fe layer on $\mathrm{Au}$, the RHEED intensity oscillation continues for a long time. On the other hand for the growth of $\mathrm{Fe}$ on $\mathrm{Ag}$ the oscillation is not clearly observed. This result suggests that the Fe layer on $\mathrm{Ag}$ has a poor layer-by-layer growth different from that of Fe layer deposited on $\mathrm{Au}$, which in turn suggests that quantum well states are not formed in a $\mathrm{Fe}$ layer on Ag.

The shape of the $\epsilon_{x y}$ spectra of Fe films less than 8 $\AA$ deposited on $\mathrm{Ag}$ are different from that of Fe bulk state. This suggests that the electronic structure of Fe film less than $8 \AA$ is different from that of bulk Fe. And, in $\mathrm{Fe}$ films thinner than $8 \AA$ on $\mathrm{Ag}$, there are some structures of $\epsilon_{x y}$ in $2-3 \mathrm{eV}$. The $\epsilon_{x y}^{\prime}$ structure is thought to be related with polarized Au atoms adjacent to the Fe layer from the following two reasons: (1) Schnatterrly reported a magneto-optical transition of polarized Au atoms near the plasma edge where an optical transition between $s, p$ and $d$ bands occurs in Au [10]. (2) The $\epsilon_{x y}^{\prime}$ spectra change with Au layer thickness $(x)$ in $\AA u(20 \AA) / \operatorname{Ag}(10 \AA) / \mathrm{Fe}(3 \AA) /$ $\operatorname{Au}(x \AA) / \operatorname{Ag}(4000 \AA) / \mathrm{MgO}(100)$ films.

\section{Conclusion}

We measured the magneto-optical polar Kerr effects on (100) ultrathin Fe film deposited on $\mathrm{Au}$ or $\mathrm{Ag}$ buffer (100) surfaces. In the photon energy region between 3.5 and $4.5 \mathrm{eV}$, a new $\phi_{\mathrm{K}}$ peak appears in the $\mathrm{Fe}$ layers deposited on $\mathrm{Au}$, whereas we the new peak cannot be observed in $\mathrm{Fe}$ layers deposited on $\mathrm{Ag}$. These phenomena are thought to be closely connected with the formation of quantum well states in Fe layers. The result of the RHEED intensity oscillation suggests that $\mathrm{Fe}$ layers deposited on $\mathrm{Ag}$ do not show a good layer-by-layer growth contrary to that of $\mathrm{Fe}$ layers deposited on $\mathrm{Au}$. For this reason no quantum well states should be expected in Fe layers on Ag. The $\epsilon_{x y}^{\prime}$ spectra of Fe film less than $8 \AA$ is different from that of bulk Fe. There are some structures of $\epsilon_{x y}^{\prime}$ in $\mathrm{Fe}$ films thinner than $8 \AA$, in the range of about $2-3 \mathrm{eV}$. These structures are tentatively attributed to spinpolarized $\mathrm{Au}$ atoms adjacent to the Fe layer.

\section{References}

[1] For instance, A.J. Freeman and Ru-Quan Wu, J. Magn. Magn. Mater. 104-107 (1992) 1.

[2] S.D. Bader, J. Magn. Magn. Mater. 100 (1991) 400.

[3] Y. Suzuki, T. Katayama, K. Sato, S. Yoshida and K. Tanaka, Phys. Rev. Lett. 68 (1992) 3355.

[4] F.J. Himpsel, Phys. Rev. (B) 44 (1991) 5966.

[5] J.E. Ortega, F.J. Himpsel, G.J. Mankey and R.F. Willis, Phys. Rev. B 47 (1993) 1540.

[6] Y. Suzuki, T. Katayama, A. Thiaville, K. Sato, M. Taninaka and S. Yoshida, J. Magn. Magn. Mater. 121 (1993) 539.

[7] P.B. Johnson and R.W. Christy, Phys. Rev. (B) 6 (1972) 4370.

[8] P.B. Johnson and R.W. Christy, Phys. Rev. (B) 9 (1974) 5056.

[9] P.M. Oppeneer, T. Mayrer, J. Sticht and J. Kubler, Phys. Rev. (B) 45 (1992) 10924.

[10] S.E. Schnatterrly, Phys. Rev. 183 (1969) 664. 